\title{
WHEN CAN SIGMOIDAL DATA BE FIT TO A HILL CURVE?
}

\author{
JACK HEIDEL $^{1}$ and JOHN MALONEY ${ }^{1}$
}

(Received 4 October 1995; revised 6 July 1998)

\begin{abstract}
The Hill equation is a fundamental expression in chemical,kinetics relating velocity of response to concentration. It is known that the Hill equation is parameter identifiable in the sense that perfect data yield a unique set of defining parameters. However not all sigmoidal curves can be well fit by Hill curves. In particular the lower part of the curve can't be too shallow and the upper part can't be too steep. In this paper an exact mathematical criterion is derived to describe the degree of shallowness allowed.
\end{abstract}

\section{Introduction}

The simple chemical reaction

$$
E+S \stackrel{k_{1}}{\longrightarrow} C \stackrel{k_{2}}{\longrightarrow} E+P
$$

can be described by the Michaelis-Menten Equation

$$
V=\frac{V_{\max }[S]}{K_{M}+[S]}
$$

where $[S]$ is the concentration of substrate $S$ and $V=\left|\frac{d[S]}{d t}\right|$ is the velocity of the reaction. The response curve (1) has the characteristic concave form shown in Figure 1.

An equation of similar form, the Hill equation, is frequently used in pharmacology to describe the response of an organism or tissue as a function of drug concentration

$$
V=\frac{V_{\max }[S]^{n}}{K_{m}+[S]^{n}}
$$

where $V$ is the pharmacologic response at drug concentration $[S]^{n}, V_{\max }$ is the maximum possible pharmacologic response, $K_{M}$ is the concentration of drug at which

'Department of Mathematics, University of Nebraska at Omaha, Omaha, NE 68182

(C) Australian Mathematical Society 1999, Serial-fee code 0334-2700/99 


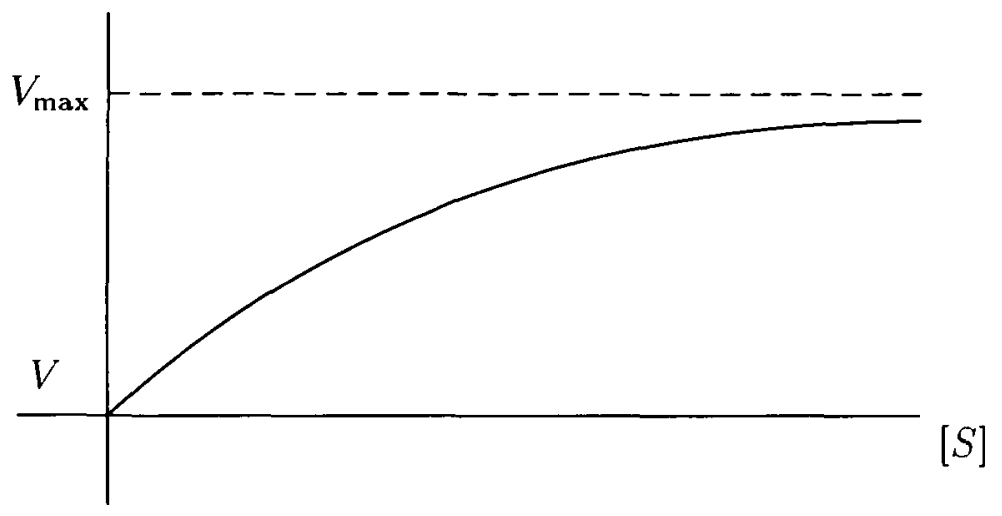

FIGURE 1.

$V=\frac{1}{2} V_{\max }$ and is also a measure of the affinity of the receptor for the drug. The parameter $n$, the Hill coefficient, can be viewed mechanistically as the number of molecules that bind to a receptor. More frequently it is regarded functionally as a shape factor determining the response curve (Figure 2).

The question investigated in this paper is related to, but distinct from, the problem of parameter identification. The Hill equation is already known to be parameter identifiable [2] which means that "perfect data" that is a complete Hill curve, uniquely determine the three parameters $V_{\max }, K_{m}$ and $n$. We are concerned with the question of which sigmoidal data curves can be fit with the Hill curves to begin with. It turns out to depend on the steepness or shallowness of the sigmoidal curve.

This is also a different question from parameter estimation where one is interested in finding the "best" Hill curve for a set of data by using some optimal least squares technique. One can always find a "best" Hill curve in some sense but we show here that it still may not be very good.

The Hill equation has three parameters and is a generalization of the MichaelisMenten equation with only two parameters. Thus it makes sense to begin with the much simpler two-parameter case.

\section{The Michaelis-Menten equation}

Even though Godfrey [1] and Godfrey and Fitch [2] have already adequately discussed the Michaelis-Menten equation, it is discussed here to introduce the methods 


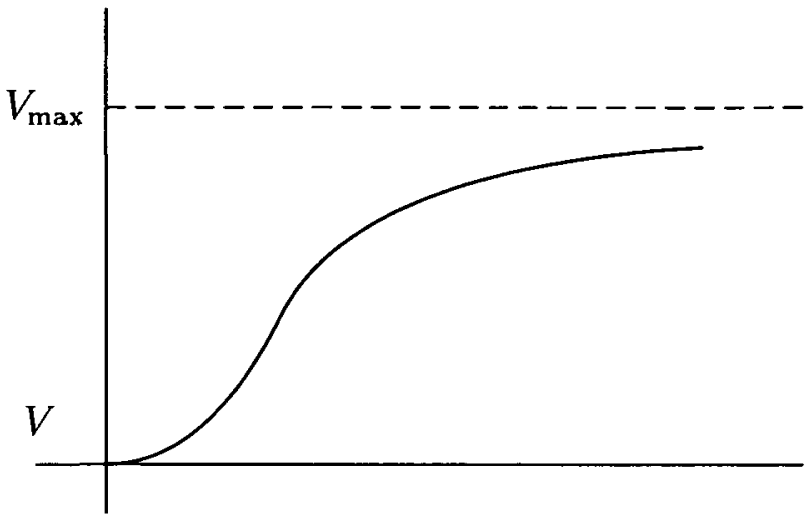

FIGURE 2.

of this paper in the simplest case. Suppose then that two data points are given:

$$
V_{1}=\frac{V_{\max }[S]_{1}}{K_{M}+[S]_{1}}, \quad V_{2}=\frac{V_{\max }[S]_{2}}{K_{M}+[S]_{2}} .
$$

Solving each equation for $V_{\max }$ yields

$$
\frac{V_{1}\left(K_{M}+[S]_{1}\right)}{[S]_{1}}=V_{\max }=\frac{V_{2}\left(K_{M}+[S]_{2}\right)}{[S]_{2}} .
$$

Now solving for $K_{M}$ gives

$$
K_{M}=\frac{\left(V_{2}-V_{1}\right)[S]_{1}[S]_{2}}{V_{1}[S]_{2}-V_{2}[S]_{1}}
$$

provided that $V_{1}[S]_{2}-V_{2}[S]_{1} \neq 0$, that is, that

$$
\frac{V_{1}}{[S]_{1}} \neq \frac{V_{2}}{[S]_{2}} \text {. }
$$

Since $V_{1} /[S]_{1}$ and $V_{2} /[S]_{2}$ are the slopes of lines from the origin to the data points, it is clear from Figure 1 that (3) holds.

It has been shown that two points on the concave curve (1) determine the parameters $V_{M}$ and $K_{M}$ uniquely, and thus the Michaelis-Menten curve (1) uniquely determines $V_{M}$ and $K_{M}$ in every case.

The conditions (3) can be replaced by

$$
\frac{V_{1}}{[S]_{1}}>\frac{V_{2}}{[S]_{2}}, \quad[S]_{1}<[S]_{2}
$$


by taking into account the concave shape of the curve (2). The inequality (4) or its reverse turns out to be significant for the investigation of the Hill equation to be discussed now.

It should be noted that the same approach which will be used to analyze the Hill equation can also be used to discuss a fractal version of the Michaelis-Menten equation [3].

\section{The Hill equation}

Since there are three parameters, $V_{M}, K_{M}$, and $n$, in (2), three data points are needed. Changing notation for simplicity in the lengthy calculation to follow, take $\left(x_{i}, y_{i}\right), i=1,2,3$, as three points satisfying

$$
y_{i}=\frac{M x_{i}^{n}}{Q+x_{i}^{n}},
$$

where $1<n, 0<x_{1}<x_{2}<x_{3}$ and $0<y_{1}<y_{2}<y_{3}$. The following theorem will be established.

THEOREM 1. Let

$$
d=\frac{\ln x_{3}-\ln x_{1}}{\ln x_{2}-\ln x_{1}}>1
$$

Then the Hill equation (5) has a unique solution for $M, Q$, and $n$ if

$$
y_{1}^{d-1} y_{3}<y_{2}^{d}
$$

and has no solution if

$$
y_{1}^{d-1} y_{3} \geq y_{2}^{d} .
$$

The proof of this theorem is computational. We may eliminate $n$ between the three equations given by substituting $\left(x_{i}, y_{i}\right)$ in (5) for $i=1,2,3$ to derive

$$
\ln Q=\frac{\frac{\ln x_{i}}{\ln x_{1}}\left(\ln \frac{y_{1}}{M-y_{1}}\right)-\ln \frac{y_{i}}{M-y_{i}}}{1-\frac{\ln x_{i}}{\ln x_{1}}}, \quad i=2,3 .
$$

Equating these two values and rearranging gives

$$
\frac{\ln x_{3}-d \ln x_{2}}{\ln x_{1}}=-\frac{\ln x_{3}-\ln x_{2}}{\ln x_{2}-\ln x_{1}}=1-d .
$$


Thus

$$
\ln \frac{y_{3}}{M-y_{3}}=(1-d) \ln \frac{y_{1}}{M-y_{1}}+d \ln \frac{y_{2}}{M-y_{2}}
$$

so that

$$
\frac{1}{y_{3}}\left(M-y_{3}\right)=\frac{y_{1}^{d-1}\left(M-y_{2}\right)^{d}}{y_{2}^{d}\left(M-y_{1}\right)^{d-1}} \text {. }
$$

Defining the functions

$$
f(M)=\frac{1}{y_{3}}\left(M-y_{3}\right) \quad \text { and } \quad g(M)=\frac{y_{1}^{d-1}\left(M-y_{2}\right)^{d}}{y_{2}^{d}\left(M-y_{1}\right)^{d-1}},
$$

it follows that (6) has a solution for $M$ wherever the graph of $f(M)$ and $g(M)$ intersect. Since the graph of $f(M)$ is a straight line, the number of intersections of $f$ and $g$ depends on the character of the function $g(M)$. The function $g(M)$ is monotone increasing and concave up. We have

$$
g^{\prime}(M)=\frac{y_{1}^{d-1}\left(M-y_{2}\right)^{d-1}}{y_{2}^{d}\left(M-y_{1}\right)^{d}} M-y_{2}+d\left(y_{2}-y_{1}\right)>0 .
$$

Likewise

$$
g^{\prime \prime}(M)=\frac{y_{1}^{d-1}\left(M-y_{2}\right)^{d-2}}{y_{2}^{d}\left(M-y_{1}\right)^{d+1}} d(d-1)\left(y_{2}-y_{1}\right)^{2}>0 .
$$

There are now several cases to consider. Suppose first that

$$
\frac{1}{y_{3}}>\frac{y_{1}^{d-1}}{y_{2}^{d}} .
$$

Then $g(M)<f(M)$ for large $M$, while $g\left(y_{3}\right)>f\left(y_{3}\right)=0$, and so by convexity of $G$ a unique point of intersection exists.

Now suppose that the other case occurs, namely

$$
\frac{1}{y_{3}} \leq \frac{y_{1}^{d-1}}{y_{2}^{d}}
$$

To proceed further the asymptote as $M \rightarrow \infty$ for $g(M)$ is needed. This is accomplished by finding the Taylor expansion of $g(M)$ for large $M$. To do this write

$$
\frac{\left(M-y_{2}\right)^{d}}{\left(M-y_{1}\right)^{d-1}}=\frac{\left(R+\left(y_{1}-y_{2}\right)\right)^{d}}{R^{d-1}}
$$


where $R=M-y_{1}$. Let $z=1 / R$ and

$$
G(z)=\left(\frac{1}{z}+\left(y_{1}-y_{2}\right)\right)^{d}=\left(\frac{1+\left(y_{1}-y_{2}\right) z}{z}\right)^{d} \quad \text { as } z \rightarrow 0 .
$$

Then $H(z)=\left(1+\left(y_{1}-y_{2}\right) z\right)^{d}$ satisfies $H(0)=1$ and $H^{\prime}(0)=d\left(y_{1}-y_{2}\right)$. Thus

$$
\begin{gathered}
H(z)=1+d\left(y_{1}-y_{2}\right) z+\cdots \quad \text { as } z \rightarrow 0, \\
G(z)=\frac{H(z)}{z^{d}}=\frac{1}{z^{d}}+d\left(y_{1}-y_{2}\right) z^{1-d}+\cdots \quad \text { as } z \rightarrow 0,
\end{gathered}
$$

and

$$
\begin{aligned}
& \frac{\left(R+\left(\left(y_{1}-y_{2}\right)\right)\right)^{d}}{R^{d-1}}=R+d\left(y_{1}-y_{2}\right)+\cdots \quad \text { as } R \rightarrow \infty, \\
& \frac{\left(M-y_{2}\right)^{d}}{\left(M-y_{1}\right)^{d-1}}=M-y_{1}+d\left(y_{1}-y_{2}\right)+\cdots \quad \text { as } M \rightarrow \infty .
\end{aligned}
$$

Thus

$$
g(M)=\frac{y_{1}^{d-1}\left(M-y_{2}\right)^{d}}{y_{2}^{d}\left(M-y_{1}\right)^{d-1}}=\frac{y_{1}^{d-1}}{y_{2}^{d}}\left[M-y_{1}+d\left(y_{1}-y_{2}\right)\right]+\cdots
$$

Thus $d\left(y_{2}-y_{1}\right)+y_{1} \leq y_{3}$ or $d \leq \frac{y_{3}-y_{1}}{y_{2}-y_{1}}$ means $g(M)$ and $f(M)$ do not intersect.

It will be shown that the condition

$$
d>\frac{y_{3}-y_{1}}{y_{2}-y_{1}}
$$

is incompatible with $y_{2}^{d} \leq y_{1}^{d-1} y_{3}$ which is equivalent to (7). This is accomplished by examining the condition

$$
\frac{\ln x_{3}-\ln x_{1}}{\ln x_{2}-\ln x_{1}}=d=\frac{y_{3}-y_{1}}{y_{2}-y_{1}}
$$

Consider the curve $\alpha\left(x_{2}\right)=y_{2}=\left(y_{3}-y_{1}\right)\left(\frac{\ln x_{2}-\ln x_{1}}{\ln x_{3}-\ln x_{1}}\right)+y_{1}$. Then $\alpha\left(x_{1}\right)=y_{1}$, $\alpha\left(x_{3}\right)=y_{3}$, and $\alpha(x)$ is concave down $(\alpha(x)$ is a logarithmic function).

The fact that $g(M)$ and $f(M)$ do not intersect if $d \leq\left(y_{3}-y_{1}\right) /\left(y_{2}-y_{1}\right)$ means that there is no solution for points $\left(x_{2}, y_{2}\right)$ below (or on) the graph of $\alpha(x)$. It will now be shown that condition (7) means that $\left(x_{2}, y_{2}\right)$ must lie below the graph of $\alpha(x)$. We thus consider the equation $y_{2}^{d}=y_{1}^{d-1} y_{3}$, that is,

$$
y_{2}=y_{1}^{(d-1) / d} y_{3}^{1 / d} \text {. }
$$


Now

$$
\begin{aligned}
\frac{d-1}{d} & =\frac{\ln x_{3}-\ln x_{2}}{\ln x_{3}-\ln x_{1}} \quad \text { and } \\
\frac{1}{d} & =\frac{\ln x_{2}-\ln x_{1}}{\ln x_{3}-\ln x_{1}} .
\end{aligned}
$$

Thus we have the function

$$
\beta\left(x_{2}\right)=y_{2}=y_{1}^{\frac{\ln n_{3}-\ln x_{2}}{\ln n_{3}-\ln _{1}+1}} y_{3}^{\frac{\ln n_{2}-\ln x_{1}}{\ln _{3}-\ln x_{1}}}
$$

to compare with the function $\alpha\left(x_{2}\right)$. Again note that $\beta\left(x_{1}\right)=y_{1}$ and $\beta\left(x_{3}\right)=y_{3}$, and we want to show that $\beta\left(x_{2}\right)<\alpha\left(x_{2}\right)$ for $x_{1}<x_{2}<x_{3}$. Now $\alpha\left(x_{2}\right)$ can be written as

$$
\alpha\left(x_{2}\right)=y_{3}\left(\frac{\ln x_{2}-\ln x_{1}}{\ln x_{3}-\ln x_{1}}\right)+y_{1}\left(\frac{\ln x_{3}-\ln x_{2}}{\ln x_{3}-\ln x_{1}}\right) .
$$

Thus $\beta\left(x_{2}\right)<\alpha\left(x_{2}\right)$, which we want to verify, can be written as

$$
y_{1}^{\frac{\ln x_{3}-\ln x_{2}}{\ln y_{3}-\ln x_{1} 1}} y_{3}^{\frac{\ln n_{1}-\ln x_{1}}{\ln x_{3}-\ln x_{1}}}<y_{1}\left(\frac{\ln x_{3}-\ln x_{2}}{\ln x_{3}-\ln x_{1}}\right)+y_{3}\left(\frac{\ln x_{2}-\ln x_{1}}{\ln x_{3}-\ln x_{1}}\right) \text {, }
$$

or equivalently

$$
\left(\frac{y_{1}}{y_{3}}\right)^{\frac{\ln \cdot x_{3}-\ln x_{2}}{\ln x_{3}-\ln x_{1}}}<\left(\frac{y_{1}}{y_{3}}\right)\left(\frac{\ln x_{3}-\ln x_{2}}{\ln x_{3}-\ln x_{1}}\right)+1-\frac{\ln x_{3}-\ln x_{2}}{\ln x_{3}-\ln x_{1}} .
$$

Since $0<y_{1}<y_{3}$ and $0<x_{1}<x_{2}<x_{3}$, this can be expressed as

$$
y^{x}<y x+1-x \text { or } 0<y x+1-x-y^{x} \text {. }
$$

Let $\gamma(x)=y x+1-x-y^{x}$ and note that $\gamma(0)=0$ and $\gamma(1)=0$. We want to show that $\gamma(x)>0$ for all $0<x<1$. Now

$$
\gamma^{\prime}(x)=y-1-\ln y e^{x \ln y}
$$

and

$$
\gamma^{\prime \prime}(x)=-(\ln y)^{2} e^{x \ln y}<0
$$

for all $0<x<1$ and so $\gamma(x)>0$ for $0<x<1$ since $\gamma(x)$ is concave down on this interval.

This means that $\beta\left(x_{2}\right)<\alpha\left(x_{2}\right)$ for $x_{1}<x_{2}<x_{3}$. Since the inequality (7) implies that $y_{2}\left(x_{2}\right)<\beta\left(x_{2}\right)$ and, as mentioned above, there are no solutions of (6) below $\alpha(x)$, 

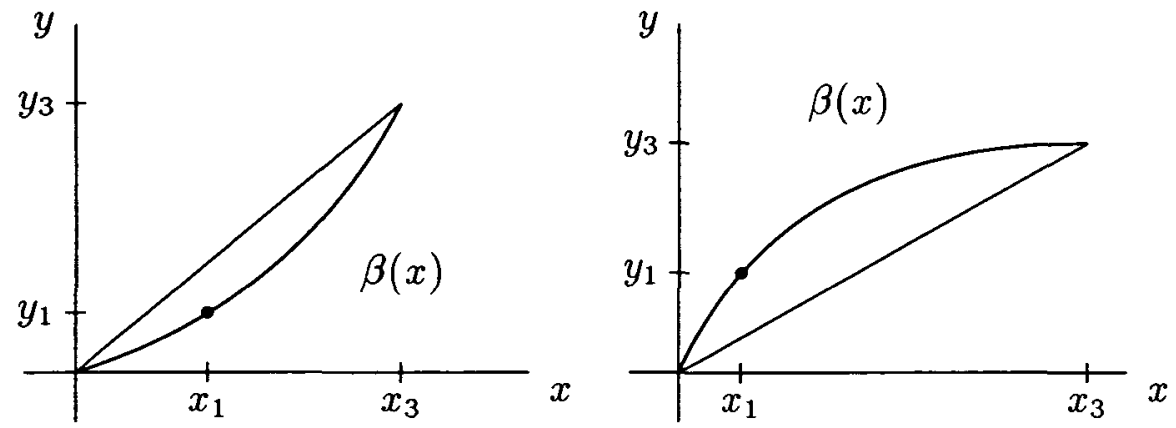

FIGURE 3.

it has finally been shown that no values of $M, Q$, and $n$ can be found when (7) holds. Theorem 1 is thus proved.

From a practical point of view it is the region where the unique solution exists which is of importance. This region is determined by the curve

$$
y_{2}\left(x_{2}\right)=y_{1}^{\frac{d-1}{d}} y_{2}^{1 / d} \text {. }
$$

The points $\left(x_{2}, y_{2}\right)$ lying above the curve (8) correspond to uniquely determined parameters $M, Q$, and $n$. The shape of (8) can be easily determined.

THEOREM 2. The curve $y_{2}\left(x_{2}\right)=y_{1}^{\frac{d-1}{d}} y_{2}^{1 / d}$ is monotone increasing, and is concave up if $y_{3} / x_{3}>y_{1} / x_{1}$, and is concave down if $y_{3} / x_{3}<y_{1} / x_{1}$.

This theorem is proven by simple differentiation of the curve (8) which is described by the function

$$
\beta(x)=y_{1}^{\frac{\ln x_{3}-\ln x}{\ln x_{3}-\ln x_{1}}} y_{3}^{\frac{\ln x-\ln x_{1}}{\ln x_{3}-\ln x_{1}}}
$$

First write

$$
\beta(x)=e^{-\frac{\ln y_{y} \ln x}{\ln x_{3}-\ln x_{1}}} e^{\frac{\ln y_{3} \ln x}{\ln x_{3}-\ln x_{1}}} y_{1}^{\frac{\ln x_{3}}{\ln x_{3}-\ln x_{1}}} y_{3}^{\frac{-\ln x_{1}}{\ln x_{3}-\ln x_{1}}} .
$$

Thus

$$
\beta^{\prime}(x)=\frac{\ln y_{3}-\ln y_{1}}{\ln x_{3}-\ln x_{1}} \frac{1}{x} \beta(x)>0
$$

and

$$
\beta^{\prime \prime}(x)=\left(\frac{\ln y_{3}-\ln y_{1}}{\ln x_{3}-\ln x_{1}}-1\right)\left(\frac{\ln y_{3}-\ln y_{1}}{\ln x_{3}-\ln x_{1}}\right) \frac{1}{x^{2}} \beta(x) .
$$




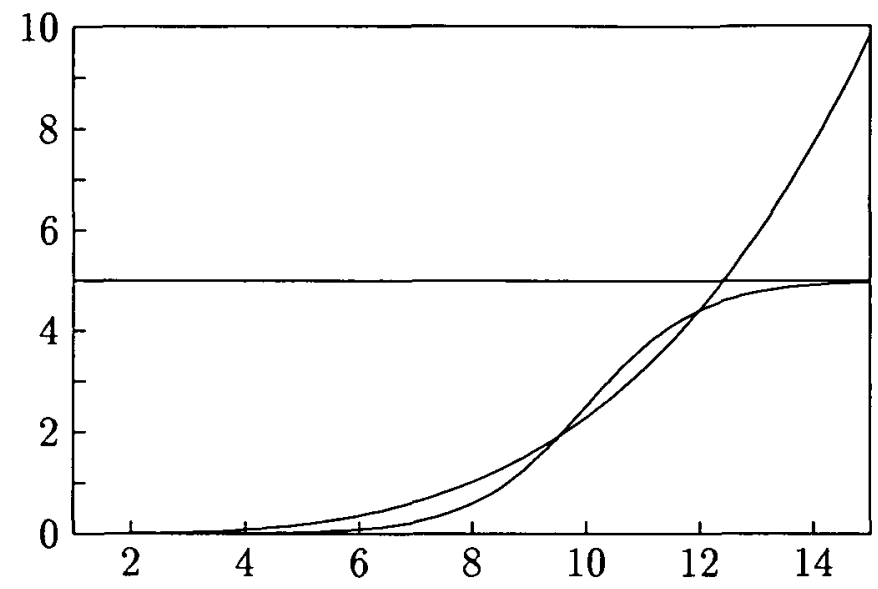

FIGURE 4.

Thus $\beta^{\prime \prime}(x)>0$ if $\left(y_{3} / y_{1}\right) /\left(x_{3} / x_{1}\right)>1$, that is, if

$$
y_{3} / x_{3}>y_{1} / x_{1},
$$

and conversely for $\beta^{\prime \prime}(x)<0$. The theorem can be illustrated as in Figure 3 .

Since the uniqueness region is above $\beta(x)$, it follows that the uniqueness region is larger if (9) holds than when the inequality goes the other way.

\section{An example}

Finally we use Theorem 1 to show that sigmoidal data can not always be fit to a Hill curve. We generate sigmoidal data by using the sigmoidal function

$$
w(x)=\frac{5}{1+e^{10-x}}-\frac{5}{1+e^{10}},
$$

which satisfies the conditions $w(0)=0$ and $\lim _{x \rightarrow \infty} w(x)=4.99$ as shown in Figure 4. Now adjust the function $y=y_{1}^{1-1 / d} y_{3}^{1 / d}$ so that it passes through the points $(1, w(1))$ and $(12, w(12))$ to obtain the concave (up) curve

$$
y(x)=w(1)^{1-\frac{\ln x}{\ln 12}} w(12)^{\frac{\ln x}{\ln 12}}
$$

also shown in Figure 4.

On the interval $(1,12)$ only points above $y(x)$ can be fit to a Hill curve passing through the points $(1, w(1))$ and $(12, w(12))$. Since $w(x)$ lies below $y(x)$ on the interval $(1,9)$, then the sigmoidal data $w(x)$ can't be fit by a Hill curve on the interval $(1,12)$. 


\section{Acknowledgement}

The authors thank the referee for many helpful suggestions.

\section{References}

[1] K. R. Godfrey, Compartmental Models and their Applications (Academic Press, New York, 1983).

[2] K. R. Godfrey and W. R. Fitch, "The deterministic identifiability of nonlinear pharmacokinetic models", J. Pharmacokinetics and Biopharmaceutics 12 (1984) 177-191.

[3] J. Heidel and J. Maloney, "An analysis of a fractal Michaelis-Menten curve", J. Austral. Math. Soc. Ser. $B$ to appear. 